\title{
Florian Flörsheimer
}

\section{Die Bundeswehr als „modernes” Wirtschaftsunternehmen}

Der Militärapparat der Bundesrepublik wurde in den letzten 20 Jahren einer Reihe von Veränderungen unterworfen. Bei dieser „Transformation“ hat es im Wesentlichen zwei zunächst parallel zueinander verlaufende Entwicklungen gegeben. Einerseits ist eine zunehmende Orientierung hin zu Auslandseinsätzen und ein entsprechender Prozess der Umstrukturierung der Truppenteile festzustellen. Dieser Prozess setzte mit Ende des Kalten Krieges ein und entwickelt sich über die Eingliederung der Nationalen Volksarmee der DDR (NVA) in die Bundeswehr, die Eingliederung der „Armee der Einheit“ in die transatlantische und europäische Militärstruktur durch die Bildung multinationaler Korps, die Reduzierung der Truppenstärke, den Aufbau von speziell für Auslandseinsätze vorgesehenen Truppenverbänden sowie eine mehrmalige Verkürzung der Wehrpflicht und schließlich die mit der neuesten Wehrreform verbundene Einführung einer Freiwilligenarmee. Anderseits fanden neoliberale Modernisierungsmodelle Eingang in den gesamten militärischen Staatsapparat. Dies reicht von der Einführung betriebswirtschaftlicher Steuerungs- und Managementmodelle, der Beratung durch externe Unternehmen bis zum „Outsourcing", d.h. der Privatisierung von Aufgaben und Dienstleistungen in den Bereichen Versorgung, Logistik, Ausbildung, Infrastruktur. Davon profitiert nicht nur die Rüstungsindustrie, sondern ein wachsender Sicherheitsdienstleistungssektor, der neben zivilen auch militärspezifische (Personal-)Dienstleistungen anbietet. Welchen Grad die Verschränkung von militärischem „Strukturwandel“ und wirtschaftlicher „Modernisierung“ hierzulande noch annehmen kann, zeigt ein perspektivischer Blick auf die Zusammenhänge zwischen Privatisierungspolitik, Wehrreform und den einem internationalen Trend folgenden wachsenden Markt für private militärische Dienstleistungen.

\section{Privatisierung in der Bundeswehr}

Die Prinzipien des New Public Management wurden in den 1990er Jahren auch in der Bundeswehr zu einem hegemonialen Modell, das in vielen Einzelbereichen aufzufinden ist. Die Bundeswehr folgte einem allgemeinen Trend in den Armeen der NATO-Staaten. Deutlichster Ausdruck war die Einführung verschiedener Privatisierungsmodelle (vgl. Petersohn 2006). Ein wesentlicher 
Schritt in dieser Entwicklung war der 1999 von der damaligen Bundesregierung mit der (Rüstungs-)Industrie vereinbarte Rahmenvertrag über „Innovation, Investition und Wirtschaftlichkeit in der Bundeswehr", in dessen Folge 2000 die „Gesellschaft für Entwicklung, Beschaffung und Betrieb“ (GEBB.) gegründet wurde. (vgl. BMVg 2000). Der Gesellschaft wurde zunächst zur Aufgabe gestellt, die „wirtschaftliche Optimierung“ von „nichtmilitärischen“ Teilbereichen der Bundeswehr (Logistik, Versorgung, Liegenschaften) in Form unterschiedlicher Privatisierungsmodelle zu organisieren. Sie etablierte sich als eine Konkurrenzinstitution zur bisherigen zivilen Bundeswehrverwaltung. 2002 wurden die ersten zwei Unternehmen im Bereich Logistik und Bekleidung gegründet: die „BW Fuhrpark Service GmbH“ und die „LH Bundeswehr Bekleidungsgesellschaft“. Partnerfirmen waren bei ersterer u.a. die Deutsche Bahn AG und bei letzterer die Lion Apparel Deutschland $\mathrm{GmbH}$ sowie die Hellmann Worldwide Logistics GmbH \& Co KG. Aufgrund zahlreicher Probleme bei der Umsetzung der ersten Privatisierungsprojekte (Kostenstreitigkeiten mit den Industriepartnern sowie viel Kritik aus den Reihen der Bundeswehr - die Erwartungen, dass sich durch die Privatisierungen Einsparungen ergeben würden, hatten sich kaum erfüllt) wurden die beiden Gesellschaften 2006 vom damaligen Bundesverteidigungsminister Jung aus der GEBB. herausgelöst. Parallel und teilweise unabhängig von den Privatisierungsprojekten der GEBB wurden andere Privatisierungsmodelle umgesetzt: „Eigenoptimierungen“ bestehender Teilsysteme der Bundeswehr bei denen der Staat mittels der Gründung privatrechtlich organisierter Gesellschaften Verwaltungsaufgaben übernimmt, Projekte der „Vollprivatisierung“ sowie unterschiedlicher „Public-PrivatePartnership“- Modelle. Dabei lassen sich drei Formen unterscheiden: das „Betreiber“-, das „Betriebsführungs“- und das „Kooperationsmodell“ (vgl. Petersohn 2006: 16f.). Beim „Betreibermodell“ stellt der private Unternehmer dem Staat Infrastruktur zur Verfügung, deren Nutzung ihm dann jeweils in Rechnung gestellt wird. Ein Beispiel sind die Einrichtungen der „Helicopter Flight Training Services $\mathrm{GmbH}^{\text {“, }}$ aus einem Industriekonsortium der Unternehmen CAE Elektronik, Eurocopter Deutschland GmbH, Rheinmetall Defence Electronics $\mathrm{GmbH}$ und Thales Deutschland bestehend, die von der Bundeswehr im Rahmen der Pilotenausbildung für den Hubschrauber NH 90 genutzt werden. Beim „Betriebsführungsmodell“ ist der Staat Eigentümer, aber nicht Betreiber der Einrichtung. Der private Unternehmer stellt auch hier die anfallenden Kosten dem Staat in Rechnung. Beispielhaft hierfür ist das Gefechtsübungszentrum bei Magdeburg, dessen Betrieb die Bundeswehr dem Unterstützungszentrum Altmark anvertraut hat. Dieses Firmenkonsortium, welches aus Serco GmbH, Saab Trainingssystem AB, der IndustrieanlagenBetriebsgesellschaft und dem Flensburger Fahrzeugbau besteht, übernahm das Gesamtmanagement, insbesondere Betrieb und die Betreuung der Technik, die Bürokommunikation und die Materialwirtschaft. Für die industrielle Betriebs- 
unterstützung ist seit 1 . September 2008 die Rheinmetall Dienstleistungszentrum Altmark GmbH, eine Tochterfirma von Rheinmetall Defence, verantwortlich (vgl. Rheinmetall Defence 2009). Auf diesem privat verwalteten Gelände übt die Bundeswehr, aber auch militärische Einheiten der Verbündeten, die Kampffähigkeit für Auslandseinsätze. Bevorzugtes drittes Modell ist das „Kooperationsmodell“", bei dem Bundeswehr und Unternehmen gemeinsam eine Dienstleistung erbringen, genannt sei die Kooperation der Luftwaffe mit EADS zur Instandhaltung des „Eurofighter“. EADS betreibt darüber hinaus mit der Luftwaffe ein „Systemunterstützungszentrum“. 2005 wurde ein weiteres Kooperationsmodell in Form der „Heereseinstandsetzungslogistik GmbH“ gegründet, die sich um die Instandhaltung des Heeres(waffen-)materials kümmert. Beteiligt sind hier die Rüstungsfirmen Krauss-Maffei-Wegemann, Rheinmetall Landsysteme und die Industriewerke Saar. In allen Kooperationen arbeiten Firmenangestellte, militärische und zivile Bundeswehrangehörige zusammen. Das größte Kooperationsprojekt (mehr als $6 \mathrm{Mrd}$. Euro Vertragsvolumen) ist das so genannte „Herkules“-Projekt, bei dem die gesamte zivile Informations- und Kommunikationstechnologie der Bundeswehr erneuert wird. Dieses Projekt wurde 2001 gestartet, lief aufgrund von Schwierigkeiten (Steinmetz 2005: 26) und trotz steigender Kosten jedoch erst 2006 an. Mit dem industriellen Vertragspartner, der Bietergemeinschaft SI, bestehend aus Siemens und IBM, wurde eigens dafür eine Gesellschaft, die BWI Informationstechnik gegründet. Eine Kooperation gibt es auch mit der Deutschen Post bzw. deren Tochterunternehmen DHL. Während die Bundeswehr mit der Post schon seit 1955 im Bereich der inländischen Briefzustellung („Feldpost“) zusammenarbeitet, diese seit 1992 auch auf die Lieferung in Einsatzgebiete ausgedehnt wurde, übernimmt DHL seit 2002 den nationalen wie weltweiten Transport leichter militärischer Ausrüstung bis $50 \mathrm{~kg}$. DHL, die auch für andere Militärs der EU und der NATO arbeitet und in Kriegsgebiete wie den Irak liefert, gründete eigens für solche Geschäfte eine interne Abteilung „Military Affairs Bundeswehr/NATO“. Das Unternehmen bewarb sich 2008 um einen von der Bundeswehr ausgeschriebenen milliardenschweren Zehn-Jahres-Vertrag, der die umfangreiche Lagerung und den Transport von Soldaten und Kriegsgerät in Einsatzgebiete vorsah. Im November 2009 nahm DHL aufgrund der als zu hoch eingeschätzten Risiken von der Bewerbung Abstand (vgl. Dachverband der Kritischen Aktionäre und Aktionärinnen 2010). ${ }^{1}$ Schließlich gibt es Dienstleistungen, die die Bundeswehr komplett bei privaten Anbietern einkauft. Dazu gehören die Überweisung der Grundausbildung von Piloten an die Lufthansa, die Reparatur von Marineschiffen durch private Werften oder

1 Auf der Hauptversammlung der Deutschen Post von 2010 forderten die „Kritischen Aktionärinnen und Aktionäre“ den vollständigen Rückzug aus dem Geschäft der Militärlogistik und die Abschaffung der entsprechenden Abteilung. Sie konnten sich nicht durchsetzen. 
die Bewachung von Liegenschaften durch private Sicherheitsfirmen. Auch bei Auslandseinsätzen beauftragt die Bundeswehr private Dienstleister für Aufbau, Bewachung und Betrieb ihrer Feldlager, ebenso für nichtmilitärische Dienstleistungen wie die Anstellung von Hilfspersonal für die Truppenküche, Betreuungseinrichtungen, Verkaufsstände und sanitäre Anlagen. Beispielsweise wurde die deutsche Firma Ecolog AG mit der Müllentsorgung, Betreuung und Reinigung von Sanitäranlagen in einer Kaserne in Afghanistan beauftragt oder Rheinmetall-DeTec für Wartung von Waffengerät in Afghanistan. Es handelt sich bei den von der Bundeswehr bisher beauftragten Firmen zwar nicht um die so genannten „Privaten Militärischen Unternehmen“, die mit eigenem Personal - Söldnern - Kriegseinsätze durchführen. Jedoch zeigt der gegenwärtige Trend, dass sich auch die Bundeswehr auf ganz unterschiedliche Weise mit den aufstrebenden privaten Sicherheits- und Militärfirmen wird auseinandersetzen müssen (vgl. Hutsch 2009).

\section{Private Sicherheits- und Militärfirmen in Deutschland}

Die Bundeswehr versteht sich mittlerweile als Wirtschaftsunternehmen, das im Wettbewerb mit der zivilen Wirtschaft um ,Fachkräfte' konkurriert (vgl. Attraktivitätsagenda 2011 des Deutschen Bundeswehrverbands). Daher intensiviert sie ihre Beziehungen zum Arbeitsmarkt, zu Jobagenturen, Zeitarbeitsfirmen, Handwerks- und Handelskammern. Eine wichtige Rolle spielt dabei der Berufsförderungsdienst, der zwischen Bundeswehr und Privatwirtschaft vermittelt, Aus- und Weiterbildungsmaßnahmen organisiert und finanziert, sowie Kontakte zu privaten Bildungseinrichtungen, Arbeitsagenturen und Betrieben pflegt. Dazu zählen auch der Kontakt zu Sicherheitsfirmen bzw. den entsprechenden Akademien, die die Ausbildung zu Personenschützern oder anderen Berufen mit „Sicherheitsaufgaben“ organisieren. Der Berufsförderungsdienst der Bundeswehr finanziert Weiterbildungsangebote für ausscheidende Bundeswehrsoldaten, u.a. für die Weiterbildung in Sicherheitsfirmen als „Sicherheitsberater" etc. Die Firmen sparen dadurch Kosten bei der Ausbildung, zumal sie beim Rückgriff auf Soldaten schon gut ausgebildetes Personal bekommen. Und seit vor ein paar Jahren das nationale Sicherheitsgewerbe mehrere staatlich anerkannte Berufsabschlüsse geschaffen hat und damit das Berufsbild in der „Sicherheitsbranche“ ausdifferenziert wurde, hat sich dies zusätzlich auf den privaten Aus- und Weiterbildungsmarkt fördernd ausgewirkt. Damit hat sich gerade für ehemalige Soldaten und Polizisten ein erweitertes, ziviles Berufsfeld ergeben, zumal die Branche seit ein paar Jahren ein großes Wachstum zu verzeichnen hat. Der Berufsförderungsdienst zahlt ausscheidenden Zeitsoldaten bis zu 9.146,25 Euro für die Finanzierung von solchen Maßnahmen (vgl. Sonderveröffentlichung der Ausgabe November 2010 des Magazins „Die Bundeswehr"). Dieses Angebot wurde in den letzten Jahren auch genutzt: Die Zahl der bewilligten Förderungen stieg von 2005 - 2007 um rund 80\% (von 
knapp 1000 auf knapp 1800 Soldaten). Dazu kommen noch die üblichen Geldauszahlungen an die ausscheidenden Soldatinnen und Soldaten. Schätzungsweise sollen so an die 14 Mio. Euro Steuergelder an ausbildende Sicherheitsakademien und -firmen geflossen sein (Hutsch 2009: 53f.). Maßnahmen wie diese befördern das boomende Wachstum der internationalen Sicherheitsund Militärindustrie ${ }^{2}$ auch in Deutschland. Deutsche Firmen spielen auf dem weltweiten Markt des privatisierten Krieges noch keine große Rolle, sie wollen jedoch am globalen Boom teilhaben. Daher beschäftigen Firmen wie Finger Security, Brillstein Security, Baltic Security-Network Academy, Paladin Risk, Ecolog AG, Praesidia Defence, Preatoria Beratung GmbH, Asgaard German Security Group u.a. ehemalige Bundeswehrsoldaten oder Bundespolizisten (vgl. Hutsch 2009: 39f; Behördenspiegel Oktober 2009: 63) zunehmend auch für Aufträge in Kriegsgebieten. Offiziell behaupten die Firmen, ihre Mitarbeiter nicht für Kampfeinsätze einzusetzen, sondern „nur“ für (bewaffneten) Personenschutz, militärische Beratung und Ausbildung. 2010 wurde öffentlich, dass die Firma Asgaard German Security Group plane, an die 100 ehemalige Bundeswehrsoldaten nach Somalia zu schicken, um einen dortigen Politiker zu schützen (vgl. Feyder/Richter 2010). Dieser Fall machte - ebenso wie der Fall der Firma BDB Protection GmbH, die 2007 ihre Dienstleistungen nach Libyen verkaufte - deutlich, wie sehr die privaten Sicherheitsfirmen über Netzwerke mit der Bundeswehr in Beziehung stehen. Nicht selten handelt es sich bei Firmeninhabern und Personal um Reservisten der Bundeswehr. Es wird nicht kontrolliert, welchen Tätigkeiten deren Personal im Ausland nachgeht. Die Firmen tragen dazu bei, dass auch aus Deutschland professionelles Personal für den internationalen Markt zu Verfügung steht. ${ }^{4}$

Auf Söldner und private Sicherheitsfirmen bezogene Bestimmungen im Völkerrecht, aber auch im deutschen Recht, sind derzeit völlig unzureichend, um vor allem im Ausland tätige private Sicherheitsdienste strafrechtlich zu verfolgen. Auf internationaler Ebene gibt es neben einigen wenig verbindlichen Vereinbarungen die „Anti-Söldner-Konvention“ der UNO bzw. eine Söldnerdefinition im „II. Zusatzprotokoll“ der Genfer Konventionen. Dort ist der Söldner-Begriff

2 Die Zahl weltweit tätiger „Mitarbeiter“ dieser Firmen - besser ausgedrückt: der Söldner wird auf über eine Million geschätzt, die Mehrheit von ihnen sind ehemalige Soldaten staatlicher Armeen. Die Zahl der Militärfirmen wird auf ungefähr 1000 geschätzt. Mittlerweile pendelt der jährliche Umsatz der Branche zwischen 170 und 200 Mrd. Euro, je nachdem, welche Dienstleistungen im Einzelnen hinzugerechnet werden Dominiert wird der Markt von US-amerikanischen und britischen Firmen wie DynCorp, ArmorGroup International oder Aegis Defence Services. (vgl. Hutsch 2009).

3 Diese Tätigkeiten lassen sich keineswegs sauber von Kriegsbeteiligungen trennen. Tatsache ist jedoch, dass die Firmen so gut wie keiner staatlichen Kontrolle unterliegen.

4 In vielen Zeitschriften der Bundeswehr sowie in anderen Militärfachzeitschriften finden sich eine Vielzahl von Werbungen für die Ausbildung und Tätigkeiten im ,zivilen' Sicherheitsbereich sowohl von privaten Schulen wie von Sicherheitsfirmen. 
aber so eng definiert, dass es bisher ein Leichtes für die Firmen war, sich davon abzugrenzen. Auf nationaler Ebene gibt es in Ländern wie beispielsweise den USA, Großbritannien oder Südafrika durchaus Gesetze, die sich explizit auf die Firmen beziehen, oder die für die Tätigkeiten der Sicherheitsfirmen relevant sind wie Kriegswaffenkontrollgesetze, Exportgesetze etc. Letztere gibt es auch in Deutschland, beziehen sich aber nicht explizit auf die Sicherheitsfirmen. Auch hier ist nur die Strafbarkeit von Individuen vorgesehen, sowie ein strafverfolgendes Interesse entweder Deutschlands oder des Landes, in dem ein Deutscher als Söldner tätig wurde. Diesem kann dann die Tätigkeit als solche nach deutschem Recht nicht zum Vorwurf gemacht werden, sondern nur nachweisbare Verbrechen. Als Reaktion auf diese Entwicklung gab es in den letzten Jahren mehrere Initiativen im Bundestag, die von einer Regulierung des Söldnerwesens bis zu einem Verbot gingen (vgl. BT-Drs. 16/10846 bzw. 16/11375). Dennoch ist bis heute weder das eine noch das andere umgesetzt worden. Es werden dabei verschiedene Gründe angeführt, beispielsweise die rechtlich umstrittene Frage, ob ehemalige Bundeswehrsoldaten im Sinne des § 109h StGB (Anwerben für fremden Wehrdienst) straffällig werden, wenn sie von einer privaten Militärfirma für den Einsatz im Ausland angeworben werden. Dazu kommt, dass im deutschen Recht der Begriff „militärische Tätigkeit“ nicht definiert ist. Neuere internationale Vereinbarungen wie das so genannte „Montreux-Abkommen" von 2008, das in Folge der vielen Skandale um private Sicherheits- und Militärfirmen auf den Kriegsschauplätzen des letzten Jahrzehnts zustande kam, hatte als Ergebnis nur die Aufstellung eines relativ unverbindlichen bzw. wirkungslosen internationalen Verhaltenskodexes zwischen den wenigen beteiligten Ländern, NGOs und dem internationalen Verband privater Sicherheitsfirmen (IPOA seit Ende 2010 ISOA), dem nur ein paar Dutzend der großen angloamerikanischen Firmen angehören. Es handelt sich hierbei hauptsächlich um Selbstverpflichtungserklärungen. Staatliche Sanktionsmaßnahmen, die auch erwähnt werden, sind abhängig von nationaler Kodifizierung. Die deutsche Bundesregierung hat aber bisher keine der dort beschriebenen Maßnahmen in nationales Recht umgesetzt (Hutsch 2009: 38f; vgl. Feyder/Richter 2010). Das auch den Militär- und Sicherheitsfirmen zugestanden Primat des „freien Unternehmertums“ wird trotz besseren Wissens über die Gefahren, die von den privaten Firmen und ihren Söldnern ausgehen - die grundsätzliche Gefahr einer Untergrabung des staatlichen Gewaltmonopols, die internationale Verletzung von Menschenrechten - höher gewertet (vgl. Uessler 2006: 204f.).

\section{Struktur- und Wehrreform}

Im „Bericht der Strukturkommission der Bundeswehr“ von Oktober 2010 wurden zahlreiche Vorschläge ${ }^{5}$ zu einer umfassenden Reform der Bundeswehr

5 Auf der Basis des Berichts der Kommission wiederum soll die konkrete Ausarbeitung der 
vorgestellt. ${ }^{6}$ Wesentliches Merkmal ist eine an Zentralisierung, „Konzentration auf Kernfähigkeiten“, „Effizienz“ und Kaderisierung orientierte Rationalisierungsstrategie. Neben der Abschaffung der Wehrpflicht, einer radikalen Verkleinerung des Bundesministeriums der Verteidigung um ca. die Hälfte der Dienstposten sowie der kompletten Verlagerung des Ministeriums nach Berlin, stehen die Einführung und Standardisierung betriebswirtschaftlicher Kontrollund Steuerungsverfahren, eine Neuordnung des Verhältnisses der Bundeswehr zur Rüstungsindustrie, Veränderungen in der Rekrutierungspraxis und der zivilen wie militärischen Beschäftigungsverhältnisse und die Aufwertung des Reservistenstatus auf dem Programm (Bericht der Strukturkommission 2010). Wesentlich ist auch die Straffung und Verringerung der Zahl der militärischen Kommandostrukturen vom Ministerium bis zur Ebene der Einsatzkommandos. Verfahrensabläufe sollen vereinfacht, verantwortliche Stellen gebündelt und bisherige Hierarchieebenen im zivilen wie im militärischen Bereich abgebaut werden. Durch die Reduzierung von Dienstposten in Stäben und militärischen Verwaltungsstellen werden mehr Soldaten für Kampfeinsätze freigestellt. Für bestimmte übergreifende Fähigkeiten und Funktionen der Teilstreitkräfte werden gemeinsame „Fähigkeitskommandos“ oder „Kompetenzzentren“ gegründet. Die Kommandogewalt wird auf vier Ebenen einer Befehlskette beschränkt: den Bundesminister, den Generalinspekteur, den Befehlshaber des Einsatzführungskommandos und den Befehlshaber der Einsatzkräfte vor Ort. Schließlich soll der Generalinspekteur „Oberkommandierender der Streitkräfte (Chief of Defence)“ werden (31). Das Ministerium wird von Grund auf neu konzipiert und auf einige als wesentlich erachtete Aufgaben, wie beispielsweise die zentrale Führung und Steuerung der untergeordneten militärischen Bereiche, beschränkt. Die Zahl der zivilen und die militärischen Abteilungen des Ministeriums wird dabei von 17 auf 8 verringert: „Strategie/Planung“, „Einsatz“, „Struktur/Organisation/Ausbildung“, „Unterstützung“, „Personal“, „Material“, „Haushalt“ und eine Abteilung „Zentralbereich“. In der Abteilung „Unterstützung“ beispielsweise wird die Stelle eines IT-Direktors geschaffen, welche der Abteilungsleiter wahrnehmen soll. Die Abteilung soll für die Betreuung im Sinne einer „IT-Governance“ der gesamten IT der Bundeswehr zuständig sein und externe IT-Berater hinzuziehen können. Dem Bundesminister selbst werden drei weitere Abteilungen direkt unterstellt: die Abteilung „Policy/Militärpolitik“, „Presse- und Informationsstab“ sowie eine Abteilung „Bundeswehr-Controlling“.

Der gesamten Bundeswehrverwaltung werden betriebswirtschaftliche Berechnungs- und Kostenmodelle vorgeschrieben; „Staatliche Doppik“ und „Budgetierung" sind für fast alle Ebenen gleichermaßen vorgesehen. Entsprechend

Umsetzung der Vorschläge erfolgen.

6 Mittlerweile (Januar 2011) ist die Umsetzung dieser Pläne beschlossene Sache. 
diesen Vorstellungen werden in der Regel zwischen politischer Leitung und den einzelnen Bereichen zu erbringende „Fähigkeiten“ und zu erfüllende „Ziele" definiert. Zur Kontrolle dieser Vereinbarungen sowie der Bewertung der Ergebnisse wie der entstandenen Kosten wird ein - davor wesentlich nur auf den Rüstungs- und Kooperationsbereich beschränktes Instrument - nun noch stärker auf die gesamte Bundeswehrverwaltung angewendet, das so genannte „Bundeswehr-Controlling“ (62). Hierbei beruft sich die Bundeswehr auf frühere Reformerfahrungen in der öffentlichen Verwaltung und in der Praxis privater Großkonzerne. Einer zentralen Kontrolleinheit, die bei der politischen Führung angesiedelt ist, wird künftig zur Aufgabe gemacht, die politischökonomische Funktionsfähigkeit des Gesamtsystems „Bundeswehr“ gegenüber nachgeordneten Bereichen zu überwachen. Dabei ist die Umsetzung der Kontrolle politischer Vorgaben für die Bundeswehr über ein neues Informationssystem vorgesehen, durch das wiederum möglichst viele Informationen aus allen Teilen der Bundeswehr wie aus den Einsätzen zentral bei der politischen wie der militärischen Leitung ohne Verzögerungen durch Zwischenebenen zusammengeführt werden (33). Aufgabe dieser Kontrolleinrichtungen ist außerdem, Auftrags- und Leistungsinhalte für die wichtigen Einheiten und Prozessabläufe als „Produktivitätsziele“ zu definieren. Eingesetzt werden „moderne Controllinginstrumente“ wie „Lebenszykluskostenbetrachtungen“, „Investitionsrechnungen“ und „Aufwand/Nutzen-Bewertungen von Einsatzszenarien“. Sämtliche Aufgaben, die nicht die wesentlichen militärischen Aufgaben berühren, werden daraufhin überprüft, ob sie zwecks Kostenreduktion ausgelagert werden können. Die Überwachung durch betriebswirtschaftliche Kontroll- und Steuerungsmodelle ist gleichfalls für den Prozess der Strukturreform selbst vorgesehen. Entscheidungen der Leitung werden über ein zentrales „Projektmanagement“ und dessen Leiter, der mit weitgehenden Kompetenzen ausgestattet ist, in operative Maßnahmen übersetzt. Dieses „Projektmanagement“ betreut Steuerung, Koordination und die Verbindung der einzelnen Arbeitsschritte wie das „Projektcontrolling“, die Bearbeitung funktionaler Querschnittsthemen, die Interdependenzen zwischen Organisationseinheiten sowie die „Kommunikation“ der Transformation.

Der Erfolg der Transformation wird durch Definition konkreter „Zielgrößen“, die in einer vorgegebenen Zeit erreicht und über ein „unabhängiges Controlling" gemessen werden, messbar gemacht. So ist beabsichtigt, ein externes Beratungsgremium einzusetzen, das als Projektorganisation die Umstrukturierung von Ministerium und Bundeswehr begleiten und die Beschlüsse der politischen Leitung unterstützen soll. Da die Bundeswehr zu einer, verschlankten', hochbeweglichen und technisch komplexen Einsatztruppe werden soll, bedarf sie des schnellen und unkomplizierten Zugriffs auf passendes Waffen- und sonstiges Gerät. Dementsprechend wird der „Beschaffungsprozess“ erneuert, die Rüstungsproduktion auf die Bedürfnisse der Bundeswehr für ihre Einsätze 
orientiert und den Herstellern durch einen „kooperativen Beschaffungsprozess Planungssicherheit gegeben“. Für diese Zwecke ist die Gründung einer „Beschaffungsagentur" vorgesehen, die externe Berater heranziehen kann (37). Schließlich sei erforderlich, die vorhandene Arbeitsteilung zwischen der Bundeswehr und der Wirtschaft im Sinne der Einsatzorientierung zu vertiefen und nicht einsatzrelevante Prozesse weiter zu privatisieren.

Nicht nur die Organisationsstruktur der Bundeswehr, auch Rekrutierung, Verwendung und die Mittel der Bindung des militärischen wie des zivilen Personals werden reorganisiert. Zur Kompensation des mit dem Wegfall der Wehrpflicht bisher verbundenen Vorteils, bei der Rekrutierung von Zeit- und Berufssoldatinnen zu einem wesentlichen Teil auf Wehrpflichtige zurückgreifen zu können, sowie des wegfallenden Zivildienstes wird ein freiwilliger (Wehr-) dienst eingeführt. Obwohl Musterung und Einberufung damit wegfallen, wird zur Erleichterung der Werbung um RekrutInnen die Erfassung der potenziell wehrfähigen Männer und Frauen beibehalten. Der freiwillige Dienst sieht eine ein- bis maximal zweijährige Verpflichtung vor, die neben dem militärischen Dienst in der Bundeswehr auch in zivilen Bereichen wie der Pflege oder dem Katastrophenschutz geleistet werden kann. Beabsichtigt wird damit, für die Vielzahl weiterhin notwendiger, als gering qualifiziert geltenden Arbeiten kostengünstig entsprechendes Dienstleistungspersonal zu bekommen. Für den Dienst in der Bundeswehr wird von den Freiwilligen zusätzlich verlangt, sich für mindestens 15 Monate zur „Teilnahme“ an Auslandseinsätzen zu verpflichten, da dafür eine entsprechende Ausbildung erforderlich ist. Diese neue Situation führt dazu, dass sich die Bundeswehr einem verschärften Wettbewerb um gut ausgebildeten Nachwuchs mit anderen Arbeitgebern ausgesetzt sieht. Weil sie nun ausschließlich auf freiwilliges Engagement und Interesse angewiesen ist, konkurriert sie mit anderen zivilen Arbeitgebern um qualifiziertes Personal, d.h. „Fachkräfte“, und muss daher entsprechende Lockangebote machen. Da die gesamte Bundeswehr mit Nachdruck auf Auslandseinsätze ausgerichtet wird, der gleichzeitig zur Verfügung stehende Personalumfang jedoch begrenzt ist, steht die bestmögliche Nutzung des gesamten Personals für solche Einsätze im Vordergrund. Dazu gehört, das Zahlenverhältnis des militärischen Einsatzpersonals zum zivilen wie militärischen Unterstützungspersonal zu seinen Gunsten zu verändern. Als entscheidend wird jedoch die Aufhebung der bisherigen Trennung zwischen Verwaltung und Streitkräften gesehen, d.h. das Personal soll grundsätzlich für beide Tätigkeitsbereiche geeignet sein. Als Voraussetzung einer entsprechenden Umsetzung dieser Vorhaben wird ein neues Verständnis der Bundeswehr als „Binnenarbeitsmarkt“ (96) gesehen, in dem das gesamte Personal trotz Statusunterschieden als einheitlicher Korpus betrachtet wird, dessen einzelne Mitglieder einer bestmögliche Verwendung zugeführt werden können. Dazu wird von allen Bundeswehrangehörigen ein „bundeswehrgemeinsames Denken“ erwartet (45). Für eine umfassende Ver- 
wendung des Bundeswehrpersonals werden eine teilweise Abkehr von traditionellen militärischen Strukturen und die Unterordnung aller Teile unter ein vorgegebenes Ziel vorausgesetzt. Dafür wird die Funktion gegenüber dem Status eines Bundeswehrangehörigen aufgewertet, beispielsweise soll sich die Vergabe von Dienstposten weniger an Status oder Dienstalter, sondern mehr an Leistung und Verwendungsfähigkeit orientieren. Trotz dieser Orientierung an der Funktion wird eine neue militärische Hierarchie etabliert, die im Wesentlichen aus drei Gruppen besteht. Die erste, die freiwilligen Wehrdienst Leistenden, sollen im In- und Ausland vor allem im Bereich von Unterstützungsmaßnahmen und einfachen Dienstleistungen eingesetzt werden. Die zweite Gruppe bilden die „Spezialisten“, d.i. speziell ausgebildete militärische, technische und medizinische Fachkräfte, die die wesentliche Gruppe für die Einsätze bilden und sich hauptsächlich aus den Zeitsoldaten und -soldatinnen rekrutieren. Die dritte Gruppen bildet schließlich die „generalistisch“ ausgebildete Führungselite, die neben militärischen vor allem Managementfähigkeiten aufweist. Entsprechend der Vorstellung vom „Binnenarbeitsmarkt“ Bundeswehr soll es möglich gemacht werden, einfacher als bisher die jeweilige Gruppe über neue Laufbahnmodelle oder „Verwendungsaufbaukonzepte“ zu wechseln. Als wichtig werden daher größere Investitionen in die Ausbildung der Soldaten und Soldatinnen wie der zivilen Mitarbeiter und Mitarbeiterinnen gesehen.

Zentrales Ziel ist die Generierung einer Multifunktionalität des Personals durch die vielseitige Ausbildung und Verwendbarkeit des gesamten militärischen und zivilen Personals, weshalb innerhalb der Bundeswehr ein problemloserer Wechsel der Ausbildung bzw. der Tätigkeiten ermöglicht werden soll (96). Deshalb wird auch angestrebt, die Ausbildung zu reformieren. Sie wird ausgebaut, stärker an wirtschaftliche Interessen geknüpft und an zivilen Berufsbildern orientiert, sowohl zur Werbung von ReservistInnen wie auch zur Qualifizierung des bestehenden Personals. Für die drei Gruppen der SoldatInnen wie den verschiedenen Zivilbeschäftigten soll es übergreifende „Projektorganisationen“, gemeinsame „Ausbildungsmodule“ oder „Verwendungsmodelle“ geben (96). Da es besonders an Personal bei „spezialisierten Verwendungen“ (42) fehle, werden für die Ausbildung von Spezialisten neue Laufbahnen geschaffen. Spezialisierte Ausbildungen wie medizinische oder ingenieurtechnische Ausbildungen werden ausgebaut, da sie als einsatzrelevant und somit entscheidend für die Bundeswehr gelten. Die akademische Ausbildung wird für Status- und Laufbahngruppen erweitert bzw. geöffnet (44). Die Ausbildung der RekrutInnen wird zum Teil privatisiert, indem mehr Verträge mit gewerblichen Bildungseinrichtungen oder ausbildenden Betrieben geschlossen werden, die Bundeswehrangehörige während und auch nach ihrer Dienstzeit ausbilden. Anvisiert wird eine intensivere Zusammenarbeit mit zivilen (Hoch-)Schulen sowie privaten Drittmittelgebern (44). Mit einer modifizierten Rekrutierungspraxis soll erreicht werden, dass der gesamte Personalkörper der Bundeswehr 
grundlegend den Bedürfnissen und Notwendigkeiten kriegerischer Auslandseinsätze angepasst wird. Im Wesentlichen beinhalten darauf bezogene Vorschläge die Verbesserung von Leistungen sowie die Schaffung neuer Anreizsysteme. Darunter fällt die Verbesserung der akademischen wie fachspezifischen Ausbildung, die Verbesserung der individuellen Verwertbarkeit dieser Ausbildung während des Dienstes wie danach im Zivilleben; dazu zählt auch die Vereinfachung von Tätigkeits- und Berufswechsel innerhalb der Bundeswehr sowie im Wechsel mit der zivilen Wirtschaft. Durch die Aufstockung sozialer wie finanzieller Leistungen werden Anreize für Eintritt und Verbleib in der Bundeswehr geschaffen. Dazu gehören die Einrichtung eines Betreuungszentrums für die zunehmende Zahl von Soldaten und Soldatinnen, die in Folge von Kriegseinsätzen an „Posttraumatischen Belastungsstörungen“ leiden, der Ausbau von Weiterverwendungsmöglichkeiten Kriegsgeschädigter in Bundeswehr und öffentlicher Verwaltung, die Wahl zwischen Trennungsgeld und Umzugsvergütung, um die Verwendungs-Mobilität der Soldat/innen zu „verbessern“, die Erhöhung von Entlassungsprämien und Versorgungsansprüchen und die Leistungen des „Beruffförderungsdienstes“. Bemerkenswert ist der Hinweis, dass alternativ zur Förderung von Weiterbildungsmöglichkeiten die Förderung durch die Auszahlung von Bargeldbeträgen vorgeschlagen wird (97). Mit einer solchen Praxis der vermehrten Barauszahlungen würde die Bundeswehr jedoch ein Instrument der Kontrolle über die zukünftigen Tätigkeiten ihrer Abgänger abgeben - was vor allem die Kontrolle über SöldnerTätigkeiten erheblich erschweren würde. Problematisch ist auch das Vorhaben, einen potenziellen Rekruten- bzw. Spezialistenmangel durch die Aufnahme von EU-Staatsbürgern oder Soldaten von NATO-Staaten auszugleichen, weil dies eine Einfallstor für Söldner darstellen kann.

Zuletzt wird den ReservistInnen eine tragende Rolle bei der Schaffung gesellschaftlicher Akzeptanz zugesprochen, indem sie einerseits als „Einsatzreserve“ dienen, anderseits als Werbeträger' fungieren und die Bundeswehr in der allgemeinen Öffentlichkeit repräsentieren sollen. ReservistInnen sollen zudem in die verschiedensten Tätigkeitsfelder der Bundeswehr stärker integriert werden, sowohl bei Auslands- wie Inlandseinsätzen, vor allem aber auch in der so genannten „zivil-militärischen Zusammenarbeit“ im In- und Ausland.

\section{Schluss}

Die Bundeswehr wandelt sich zu einer zwar verkleinerten, doch hoch spezialisierten und flexiblen Interventionstruppe, einem in die hegemoniale ökonomische Struktur eingebundenen global aktiven militärischen Unternehmen. Mit der Transformation der Bundeswehr zu einer Freiwilligenarmee ist zudem eine ,Professionalisierung' des Personals verbunden. Dies schafft im Zusammenhang mit den Maßnahmen der Strukturreform verbesserte Bedingungen für 
weitere Privatisierungen. Dadurch wird die bereits auf internationaler Ebene zu beobachtende Dynamik der gegenseitigen Durchdringung staatlichen Militärs und privater militärischer Dienstleistung verstärkt. Nicht zuletzt liegt es in der Konsequenz der Logik neoliberaler Rationalisierungsstrategien, immer mehr Dienstleistungen extern einzukaufen. Die Folge wäre ein wachsendes privates Angebot militärischer oder sich an der Peripherie militärischer Tätigkeiten befindender (Personal-)Dienstleistungen, unter der Voraussetzung, dass beispielsweise privates Kapital in der Lage ist, ausgebildete Fachkräfte von der Bundeswehr abzuwerben. Zwar scheint die Bundeswehr dem gewissermaßen zuvor kommen zu wollen, indem sie daraufhin orientiert, das selbst geschaffene Qualifikationsniveau ihres Personals auch dauerhaft für sich zu nutzen - so sollen der Anteil von Soldaten und Soldatinnen auf Zeit sowie ihre Verpflichtungszeiten erhöht werden - doch ist nicht auszuschließen, dass Personal die erworbenen (militärischen) Fähigkeiten schneller als erwünscht privat zu verwerten trachtet. Diese Tendenz wird verstärkt, wenn ausscheidenden SoldatInnen ermöglicht wird, ihre in der Bundeswehr erworbenen Fähigkeiten ohne Einschränkung in Wirtschaft und Industrie einzubringen.

Es stellt sich als weitere Frage, ob die Umsetzung der Strukturreform nicht einen erheblichen Kostenaufwand bedeutet, so dass nach Alternativlösungen gesucht werden muss, beispielsweise einer privaten Organisierung und Bereitstellung der Reservisten und Reservistinnen. Denn die Kosten der Strukturreform bzw. der eingeführten Freiwilligenarmee werden eher steigen, wie Beispiele aus anderen Ländern, die eine Freiwilligenarmee eingeführt haben - z.B. Großbritannien, Frankreich, Spanien, Dänemark, USA - zeigen. In diesen Ländern hat es nach Einführung der Freiwilligenarmee vor allem Rekrutierungsschwierigkeiten gegeben. Die Notwendigkeit, dieses Rekrutierungsdefizit auszugleichen, ${ }^{7}$ sowie die hohen Ausbildungskosten, brachten die Militärs in Schwierigkeiten, zumal in Zeiten intensiver Kriegsführung. Die von der deutschen Bundesregierung auch von dem Verteidigungsressort verlangten Sparmaßnahmen von 8,3 Mrd. Euro bis 2014 werden sich daher womöglich bald als Luftnummer erweisen. Sie stehen jedenfalls im Widerspruch zu den zu erwartenden Kosten der auf bis zu sieben Jahren veranschlagten Zeit zur Umsetzung der Struktur- und „Wehr“reform. Die Umstellung wird nicht nur in ihrer Frühphase Geld kosten, sondern auch darüber hinaus. Dies hängt natürlich auch von den militärischen Aktivitäten der Bundeswehr ab und davon, mit welchem Aufwand es der Bundeswehr gelingt, ausreichend Freiwillige zu bekommen. Diese Entwicklung birgt zudem die Gefahr, dass eine wachsende Zahl militärisch gut und vielseitig ausgebildeter ehemaliger SoldatInnen auf dem zivilen Arbeitsmarkt

7 In Großbritannien wird beispielsweise mit dem Modell des „Sponsored Reserve“ versucht, Privatfirmen an der Bereitstellung von Reservisten zu beteiligen (vgl. Petersohn 2006: 31; Krahmann 2003). 
zur Verfügung stehen wird - nicht nur für zivile Tätigkeiten oder im Bereich ziviler privater Sicherheitsdienstleistungen, sondern auch für militärische Dienstleistungen. Diese werden gegenwärtig zwar hauptsächlich von ausländischen Firmen nachgefragt, aber da sich in diesem Sektor auch profitable Geschäfte für ehemalige, betriebswirtschaftlich geschulte Führungskräfte der Bundeswehr ergeben könnten, ist denkbar, dass die Zahl deutscher Firmen, die militärische Dienstleistungen anbieten und dafür ehemalige SoldatInnen anwerben, zunehmen wird.

Da durch zahlreiche Maßnahmen in der Öffentlichkeit intensiv für die Akzeptanz und ein positives Bild der „neuen Bundeswehr“ geworben werden soll, ist zu erwarten, dass die Bundeswehr noch stärker als in der Vergangenheit in der Öffentlichkeit präsent sein wird. Wesentlich ist dabei der Versuch, die Öffentlichkeit an die Bundeswehr und ihre neue Rolle nicht nur zu gewöhnen, sondern auch Folgebereitschaft zu erzeugen und die öffentliche Wahrnehmung den Bedürfnissen der Bundeswehr anzupassen. Dem zukünftig wahrscheinlich steigenden Personalbedarf der Bundeswehr werden dabei die wirtschaftlichen Nöte oder die abnehmende Chance auf den gewünschten Studienplatz vieler junger Menschen gelegen kommen. Gerade diesen bietet die Bundeswehr dann „alternative“ Karriereaussichten, u.a. auch eine akademische Ausbildung. Aus demokratischer Perspektive bedeutet die Transformation der Bundeswehr eine relative Verschlechterung der parlamentarischen wie der öffentlichen Kontrolle sowohl über militärische Einsätze wie über die internen Verhältnisse, da die neue Struktur der politischen und militärischen Führung der Bundeswehr eine größere Entscheidungsautonomie ermöglicht. Die neue Struktur wird es diesen Führungsspitzen erlauben, sehr viel schneller militär(politische) Entscheidungen zu treffen und umzusetzen. Gleichzeitig wird der Rüstungsindustrie durch die Reorganisation des Beschaffungsprozesses und des forcierten weiteren Aufbaus von Kooperationsprojekten der Zugriff auf interne Entscheidungen der Bundeswehr erleichtert.

Schließlich kann man den gesamten Transformationsprozess als die moderate Form einer „Re-Militarisierung“ bezeichnen, der sich in einer gegenseitigen Durchdringung militärischer wie aktueller betriebswirtschaftlicher Denkweisen artikuliert, die längst zum Allgemeingut geworden sind und dadurch gesellschaftliche Durchschlagskraft besitzen. Durch die Vermischung ziviler und militärischer Tätigkeiten sowie eine höhere Durchlässigkeit bei der Personalgewinnung entwickelt die Bundeswehr „hybride“ Charakterzüge - die Gefahr besteht daher weniger in einem ausufernden neuen Militarismus alter wilhelminischer Prägung, sondern vielmehr in der schleichenden Reproduktion und Akzeptanz militärischen Denkens und Verhaltens im Alltagsbewusstsein. 


\section{Literatur}

Behörden Spiegel (2009): Aktuelle Organisation der Söldner, Oktober, 63.

- (2010): Newsletter Verteidigung, Streitkräfte und Politik, Nr. 2, 15. Oktober 2010 (letzter Zugriff 26.10.2010).

Bundesminister der Verteidigung (Hrsg.) (2000): Neuausrichtung der Bundeswehr. Grobausplanung. Ergebnisse und Entscheidungen, Bonn-Berlin.

- (2010): Leitlinien zur Ausplanung der neuen Bundeswehr, (http://www.bmvg.de/portal/a/bmvg/ ministerium?yw_contentURL=/C1256F1200608B1B/W286VBVR574INFODE/content.jsp).

Bundestagsdrucksache 16/10846 (2008): Antrag der Fraktionen der CDU/CSU und der SPD vom 12.11.2008: Nichtstaatliche militärische Sicherheitsunternehmen kontrollieren

- 16/11375 (2008): Antrag der Fraktion Die LINKE vom 16.12.2008: Internationale Ächtung des Söldnerwesens und Verbot der Erbringung militärischer Dienstleistungen durch Privatpersonen und Unternehmen.

Dachverband der Kritischen Aktionärinnen und Aktionäre (2010): Pressemitteilung Nr. 5/2010 „DHL muss sich aus der Militärlogistik zurückziehen“. Dachverband unterstützt die Initiative Zivile Post 2011.

Deutscher Bundeswehrverband (2010): Attraktivitätsagenda 2011, Bonn.

- (2010): Sonderveröffentlichung der November-Ausgabe des DBwV-Magazins „Die Bundeswehr": Aus- und Weiterbildung, Bonn.

Feyder, Franz; Richter, Alexander (2010): Deutsche Söldner für Bürgerkrieg in Somalia, http://www.tagesschau.de/inland/asgaard100.html (letzter Zugriff 26.11.2010).

Hutsch, Franz (2009): Exportschlager Tod. Deutsche Söldner als Handlanger des Krieges, Berlin.

Krahmann, Elke (2003): Controlling Private Military Companies: The United Kingdom and Germany, Paper for the International Studies Assocation Annual Convention 2003.

Petersohn, Ulrich (2006): Die Nutzung privater Militärfirmen durch US-Streitkräfte und Bundeswehr, SWP-Studie 36, Berlin.

Rheinmetall Defence (2009): Rheinmetall Defence sorgt in der Altmark für reibungslosen Betrieb des Gefechtsübungszentrums des Heeres, http://www.rheinmetall-defence.com/index.php ?fid= 5105 \&qid $=$ \&qpage $=0$ \&lang $=2$ \&query $=\mathrm{G} \% \mathrm{DCZ}$

Steinmetz, Christopher (2005): Herkules und andere Privatisierungs-Projekte. Sackgasse für die Bundeswehr? http://www.bits.de/public/pdf/querschnitt2005.pdf (Letzter Zugriff 26.10.2010).

Strukturkommission der Bundeswehr (2010): Bericht der Strukturkommission der Bundeswehr Oktober 2010. Vom Einsatz her denken. Konzentration, Flexibilität, Effizienz, Bonn.

Uesseler, Rolf (2006): Krieg als Dienstleistung. Private Militärfirmen zerstören die Demokratie, Berlin. 